\title{
Prolific Permutations
}

\author{
Michael Albert Murray Tannock* \\ Department of Computer Science \\ University of Otago \\ Dunedin, New Zealand \\ malbert@cs.otago.ac.nz mtannock@cs.otago.ac.nz \\ Submitted: Oct 21, 2020; Accepted: Mar 18, 2021; Published: Apr 9, 2021 \\ (C) The authors. Released under the CC BY-ND license (International 4.0).
}

\begin{abstract}
The concept of prolificity was previously introduced by the authors in the context of compositions of integers. We give a general interpretation of prolificity that applies across a range of relational structures defined in terms of counting embeddings. We then proceed to classify prolificity in permutation classes with bases consisting of permutations of length 2 , or 3 ; completely classifying all such classes except $\operatorname{Av}(321)$. We then show a number of interesting properties that arise when studying prolificity in $\operatorname{Av}(321)$, concluding by showing that the class of permutations that are not prolific for any increasing permutation in $\operatorname{Av}(321)$ form a polynomial subclass.
\end{abstract}

Mathematics Subject Classifications: 05C88, 05C89

\section{Introduction}

The notion of prolificity was introduced by the authors [3] in the context of compositions of integers. Briefly put, a combinatorial structure, $T$, is prolific for another structure, $P$, if every proper extension of $T$ contains more occurrences of $P$ than $T$ did (formal definitions follow below). This natural property does not seem to have been considered previously, so the next step in its exploration is to consider how it behaves in other types of structures. For the previously investigated case of compositions, the underlying structures could be thought of as equivalence relations on an ordered set in which each equivalence class is an interval. Equivalent to that original context is the class of layered permutations (which, in one line notation are represented by an increasing sequence of decreasing intervals, e.g., 215436987 corresponding to the composition 2, 3, 1, 3) and so considering prolific structures in permutation classes is a logical next step. In the next

\footnotetext{
*Supported by University of Otago Postgraduate Publishing Bursary (Doctoral)
} 


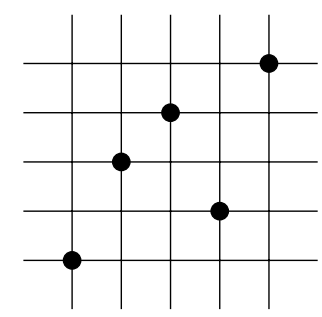

Figure 1: The plot of the permutation $\sigma=13425$.

two short sections we introduce the basic ideas and terminology of permutation classes and prolificity in general (that section can be skipped by the reader who is interested only in the concept in the context of permutation classes). Then, somewhat following the lead of Simion and Schmidt [7] (as has become the norm in considering new ideas in permutation classes) we provide a classification of prolificity in permutation classes whose bases consist of permutations of length up to 3 .

The results in this paper are largely derived and extended from the results present in Tannock's Ph.D. thesis [8]. Note that the notion of prolificity discussed here is distinct from that presented by Bevan, Homberger and Tenner [4].

\section{Permutations and permutation classes}

A permutation, $\sigma$, of size $n$ is a bijective map from $\{1 \ldots n\}$ to itself. A permutation is written in one-line notation by listing the image of each of the integers 1 to $n$ and thus the permutation $\sigma=\sigma(1) \sigma(2) \ldots \sigma(n)$. Let $\mathcal{S}_{n}$ denote the set of all permutations of size $n$, and $\mathcal{S}$ the set of all permutations of any size (we leave it to the reader to decide whether or not to include permutations of size 0 -it will not be relevant for our purposes). Denote by 1 the single permutation of size 1. An alternative viewpoint arises from considering permutations graphically, identifying each permutation $\sigma$ with its plot, that being the set of points $(i, \sigma(i))$ in the Cartesian plane.

A permutation $\tau$, referred to as the text, contains another permutation $\pi$, referred to as a pattern, if in one-line notation $\tau$ contains a subsequence of the same length as $\pi$ whose terms are in the same relative order as those of $\pi$. For example, the permutation $\tau=13425$ contains the pattern 213 by using the second, fourth, and fifth elements; however, $\tau$ does not contain the pattern 321 . Clearly containment defines a partial order on $\mathcal{S}$. If $\tau$ does not contain $\pi$ then we say that it avoids $\pi$.

We denote the containment relation by $\leqslant$. We use $\uparrow \pi$ and $\downarrow \pi$ to denote the set of permutations containing, respectively contained in $\pi$ (not necessarily properly).

A selection of elements of $\tau$ that correspond to a particular pattern, $\pi$, is called an embedding of $\pi$ in $\tau$. In this definition we are effectively identifying $\tau$ with its plot or, what amounts to the same thing, with a set of points carrying two linear orders - one which we think of as "left to right" in the plot, and the other "bottom to top". In this context the left to right order of the elements is fixed, so the range of the embedding is enough to determine the full embedding. This allows us to identify an embedding with 
its range. A permutation, $\sigma$, is said to be covered by the pattern $\pi$ if the union of all of the possible embeddings of $\pi$ into $\sigma$ is equal to the set of all elements of $\sigma$.

A permutation class, or simply class, is a set of permutations that is downwards closed in the containment order. Of course, any such class is determined by those permutations it does not contain. More specifically, given any set $B$ of permutations, the set $\operatorname{Av}(B)$ of permutations that avoid all the elements of $B$ is a class, and given any class $\mathcal{C}, \mathcal{C}=\operatorname{Av}(B)$ where $B$ is the complement of $\mathcal{C}$, or even the (containment-)minimal elements of the complement of $\mathcal{C}$ (referred to as the basis of $\mathcal{C}$ ).

Given a permutation $\sigma$ of size $n$, and a permutation class $\mathcal{C}$, the one-point extensions (in $\mathcal{C}$, which we take as understood) are the permutations of size $n+1$ in $\mathcal{C}$ that contain $\sigma$. Given a one-point extension, $\tau$ of $\sigma$ and an embedding of $\sigma$ into $\tau$, we call the point of $\tau$ that does not belong to the embedding the extension point.

The ordered inversion graph of a permutation, $\sigma$, of size $n$ is the graph on the vertex set $\{1, \ldots, n\}$ where the vertex set is ordered using the usual linear order on integers and there is an edge between the vertex $i$ and the vertex $j$ if $i<j$ and $\sigma(j)<\sigma(i)$. In the context of permutation patterns the pattern containment relation on permutations is equivalent to the induced (ordered-)subgraph relation on these ordered inversion graphs.

Given two permutations $\sigma$ and $\tau$ of length $k$ and $\ell$ respectively, the direct sum of $\sigma$ and $\tau$, denoted $\sigma \oplus \tau$, is the permutation of length $k+\ell$ defined by

$$
\sigma \oplus \tau(i)= \begin{cases}\sigma(i) & \text { if } i \leqslant k \\ \tau(i-k)+k & \text { if } k+1 \leqslant i \leqslant k+\ell\end{cases}
$$

The skew sum, $\sigma \ominus \tau$, can be obtained similarly

$$
\sigma \ominus \tau(i)= \begin{cases}\sigma(i)+\ell & \text { if } i \leqslant k \\ \tau(i-k) & \text { if } k+1 \leqslant i \leqslant k+\ell\end{cases}
$$

In the ordered inversion graph viewpoint the direct sum is obtained by appending the graph of $\tau$ to that of $\sigma$ without adding any additional edges, since no new inversions are created in the direct sum construction. Contrastingly, the graph of the skew sum is the graph of $\sigma$ followed by the graph of $\tau$ with an edge from every vertex in $\sigma$ to every vertex in $\tau$.

A permutation is said to be sum-indecomposable (respectively skew-indecomposable) if it cannot be expressed as the direct sum (respectively skew sum) of two shorter permutations. Every permutation can be uniquely expressed a sum (respectively skew sum) of sum-indecomposable (respectively skew-indecomposable) permutations called its sumcomponents (respectively skew-components). A permutation class, $\mathcal{C}$, is said to be sumclosed (respectively skew-closed) if for every pair of permutations $\sigma, \tau \in \mathcal{C}$ the permutation $\sigma \oplus \tau$ (respectively $\sigma \ominus \tau$ ) belongs to the class $\mathcal{C}$. 


\section{Prolificity in general}

We briefly visit the notion of prolificity in general combinatorial classes before specialising the concept to the context of permutations.

We suppose that we are working in some hereditary universe $\mathcal{U}$ of (finite) combinatorial structures of a particular type - fundamentally, the set of finite models of some finite relational language together with some universal theory. So, for instance, $\mathcal{U}$ could be the universe of all simple graphs, or of all simple triangle-free graphs, or of all finite sets carrying a single equivalence relation, or of all finite sets carrying a linear order and an equivalence relation whose equivalence classes are intervals (recall, this is the case of compositions mentioned in the introduction).

In such settings, there is a natural notion of embedding - an injective map from one structure to another that, co-restricted to its image, is an isomorphism. That allows us to introduce the fundamental definition:

Definition 1. A structure, $T \in \mathcal{U}$, is prolific for $P \in \mathcal{U}$, or $P$-prolific, if for all structures $T^{+}$, such that $T$ can be properly embedded into $T^{+}$, there are strictly more embeddings of $P$ into $T^{+}$than there are into $T$.

For illustrative purposes, consider this notion in the universe $\mathcal{G}$ of finite simple graphs, and $\mathcal{T}$ of finite simple triangle-free graphs. In both of these contexts note that the notion of embedding is that of induced subgraphs. In $\mathcal{G}$, every graph is prolific for the one vertex graph, and no graph is prolific for any graph with two or more vertices. The first claim is clear, and for the second observe that if a graph, $P$, is disconnected and $T$ is any graph, then extending $T$ by a single vertex adjacent to all other vertices can create no new embeddings of $P$ (and if $P$ is connected, then adding a single isolated vertex behaves similarly). However, in $\mathcal{T}$, the situation is more interesting. Since we cannot add a vertex connected to both endpoints of an edge, every triangle-free graph containing an edge is prolific for the pattern consisting of two isolated vertices.

If we consider covering structures with copies of another structure, we obtain a slight variation on the definition above, arguably somewhat more natural in the model-theoretic context. Say that $T$ is covered by $P$ if every element of $T$ belongs to the image of some embedding of $P$ into $T$.

Definition 2. A structure $T \in \mathcal{U}$ is cover-prolific for $P \in \mathcal{U}$, if every (not necessarily proper) extension of $T$ is covered by $P$.

Note that in this definition, $T$ itself must be covered by $P$. It is clear that if $T$ is cover-prolific for $P$ then it is also prolific for $P$. Moreover, if $T$ is prolific for $P$ and covered by $P$ then it is cover-prolific for $P$. It turns out that, in most natural situations, a $P$-prolific structure is necessarily covered by $P$, so there is even less variation between the definitions than it might seem at first glance.

We will retain the original definition, as it was suggested by the original motivation for this work which was a general consideration of how the number of embeddings of $P$ into a sequence of extensions could behave. 


\section{Prolificity in permutation classes}

Henceforth, we will work only in the context of permutations, and so, for the benefit of the reader who skipped the preceding section, we repeat the definition of prolificity in this context. There will generally be an ambient class $\mathcal{C}$, which we may or may not mention according to whether it seems clear from the context.

Definition 3. A permutation $\tau$ belonging to a class $\mathcal{C}$ is $\pi$-prolific if every one-point extension of $\tau$ in $\mathcal{C}$ contains strictly more copies of $\pi$ than $\tau$ did.

Equivalently, for every one-point extension, $\tau^{+}$, of $\tau$ there is an embedding of $\pi$ into $\tau^{+}$containing the extension point. There is a vacuous case where $\tau$ has no one-point extensions in $\mathcal{C}$ (i.e., $\tau$ is a maximal element of $\mathcal{C}$ ). This does not arise in the situations we will discuss, but for consistency such $\tau$ should be considered prolific for $\pi$ as the definition suggests. This would apply even if $\pi$ were not in $\mathcal{C}$, but we will always assume that to be the case.

The set of all permutations in $\mathcal{C}$ that are prolific for a given pattern $\pi$ will be denoted $\operatorname{Pro}_{\mathcal{C}}(\pi)$. Since we (almost) always work in some fixed ambient class $\mathcal{C}$, we shall frequently omit the subscript. We begin with a trivial observation (since every point in a permutation is itself a copy of $\mathbf{1}$ ).

Observation 4. Every permutation is prolific for the pattern $\mathbf{1}$, i.e., $\operatorname{Pro}_{\mathcal{C}}(\mathbf{1})=\mathcal{C}$.

Our next observation concerns the structure of $\operatorname{Pro}(\pi)$ (remember, $\mathcal{C}$ is understood, and that includes the fact that named permutations are in $\mathcal{C}$ unless specifically stated otherwise):

Proposition 5. If $\tau \in \operatorname{Pro}(\pi)$, then $\uparrow \tau \subseteq \operatorname{Pro}(\pi)$.

Proof. Let $\sigma \in \uparrow \tau$ be given and consider $\sigma^{+}$any one-point extension of $\sigma$ by a point $x$. Take any embedding of $\tau$ into $\sigma$ and consider the one-point extension of $\tau$ obtained from the relationship between this embedding and $x$. This contains a copy of $\pi$ that covers $x$ since $\tau$ is $\pi$-prolific, but interpreting this in $\sigma^{+}$shows that $\sigma^{+}$contains more copies of $\pi$ than $\sigma$ did.

Another way to put the preceding result is that the non- $\pi$-prolific permutations in $\mathcal{C}$ form a subclass of $\mathcal{C}$.

We say that $\pi$ is self-prolific if $\pi \in \operatorname{Pro}(\pi)$.

Proposition 6. If $\pi$ is self-prolific and $\mathcal{C} \cap \operatorname{Av}(\pi)$ has no maximal elements, then $\operatorname{Pro}_{\mathcal{C}}(\pi)=\uparrow \pi$.

Proof. As any permutation avoiding $\pi$ has an extension avoiding $\pi$, none of these are $\pi$-prolific. The result then follows from the preceding proposition.

Definition 7. A permutation class $\mathcal{C}$ is said to be prolifically trivial if $\pi \neq 1$ implies $\operatorname{Pro}_{\mathcal{C}}(\pi)=\{\}$. 
Lemma 8. If $\mathcal{C}$ is a permutation class such that, for every $\sigma \in \mathcal{C}$, both $\mathbf{1} \oplus \sigma$ and $\mathbf{1} \ominus \sigma$ are in $\mathcal{C}$, then $\mathcal{C}$ is prolifically trivial.

Proof. This is perhaps most easily seen by considering ordered inversion graphs. The ordered inversion graph of $\mathbf{1} \oplus \sigma$ has an initial isolated vertex, while that of $\mathbf{1} \ominus \sigma$ has an initial dominating vertex. If $\pi \in \mathcal{C}$ is of size at least 2 , and the ordered inversion graph of $\pi$ does not have an initial isolated vertex, then $\mathbf{1} \oplus \sigma$ does not contain any more copies of $\pi$ than $\sigma$ did. On the other hand, if the ordered inversion graph of $\pi$ does not have an initial dominating vertex, then $\mathbf{1} \ominus \sigma$ does not contain any more copies of $\pi$ than $\sigma$ did. Since the initial vertex of $\pi$ 's ordered inversion graph cannot be both isolated and dominating, this demonstrates that $\sigma \notin \operatorname{Pro}_{\mathcal{C}}(\pi)$, i.e., $\operatorname{Pro}_{\mathcal{C}}(\pi)=\{\}$.

There is a dihedral group of eight symmetries of $\mathcal{S}$ consisting of rotations by multiples of a right angle and reflections in the vertical, horizontal or diagonal directions. For each of these, there is a corresponding effect on the operations of sum and skew-sum. This allows us to extend the preceding lemma to the following:

Corollary 9. If a class $\mathcal{C}$ has the property that for any $\sigma \in \mathcal{C}$ both of:

- $\mathbf{1} \oplus \sigma$ and $\mathbf{1} \ominus \sigma$ or,

- $\sigma \oplus \mathbf{1}$ and $\mathbf{1} \ominus \sigma$ or,

- $\mathbf{1} \oplus \sigma$ and $\sigma \ominus \mathbf{1}$ or,

- $\sigma \oplus \mathbf{1}$ and $\sigma \ominus \mathbf{1}$.

are in $\mathcal{C}$, then $\mathcal{C}$ is prolifically trivial.

The significance of this corollary relates to the basis of a permutation class. Suppose for instance that the basis of a class $\mathcal{C}$ contains no permutations that begin (in one-line notation) with 1 . Then, for any $\sigma \in \mathcal{C}, 1 \oplus \sigma \in \mathcal{C}$. So, to avoid being prolifically trivial, the first of the conditions above implies that $\mathcal{C}$ must either have a basis element beginning with its maximum element or one beginning with its minimum element, and the last implies it must either have a basis element ending with its maximum element or one ending with its minimum element.

Putting the four conditions together, to avoid being prolifically trivial there must either be both a basis element of the form $1 \oplus \beta$ and one of the form $\gamma \oplus 1$, or one of the form $1 \ominus \beta$ and one of the form $\gamma \ominus 1$. These conditions are symmetrically related of course, so we shall assume in the sequel that the second is satisfied.

For further avoidance of trivial cases we also assume that $\mathcal{C}$ is not finite.

\section{Basis Elements in $\mathcal{S}_{2}$}

This section is included for completeness only - the results are essentially trivial. 
There are just two permutations in $\mathcal{S}_{2}$ : 12 and 21. The only non-finite class having these as basis elements and meeting our constraints is $\mathcal{I}=\operatorname{Av}(21)$ which consists precisely of the increasing permutations. Since the containment order in $\mathcal{I}$ is a chain, any $k$ elements of an $n$ element permutation constitute an embedding of the increasing permutation of size $k$ into the one of size $n$. In particular, provided that $n \geqslant k-1$ the increasing permutation of size $n$ is prolific for the increasing permutation of size $k$. Put differently, $\operatorname{Pro}_{\mathcal{I}}(\pi)$ consists of all those permutations in $\mathcal{I}$ of size at least one less than the size of $\pi$.

Note that this is a case where "prolific" and "cover-prolific" differ, since the increasing permutation of size $n-1$ is not covered by the increasing permutation of size $n$.

\section{Basis Elements in $\mathcal{S}_{3}$}

When we move on to basis elements from $\mathcal{S}_{3}$, things become a little more interesting. The set of permutations of size 3 consists of 6 elements:

$123,132,213,231,312,321$.

As a result of this there are 63 possible permutation classes defined with non-empty bases consisting of permutations in $\mathcal{S}_{3}$. However, after accounting for our conditions that the class not be finite, have at least one basis element beginning with 3 and one ending with 1 , and taking symmetry into account, only 12 classes remain, which we consider below.

\section{1 $\operatorname{Av}(321,312,213,231,132)$ and $\operatorname{Av}(312,213,231,132)$}

These two classes both have eventually constant enumeration. However, when considering prolificity it often makes sense to think of the structure of an element in a class.

The class $\operatorname{Av}(321,312,213,231,132)$ consists of all of the increasing permutations as well as the permutation 21, and as such there is one permutation of each size greater than 2. Its behaviour is fundamentally the same as the class of increasing permutations.

The other class $\operatorname{Av}(312,213,231,132)$ is the union of the class of increasing permutations and the class of the decreasing permutations. There are two permutations of each size in this class for all sizes greater than one. We already know that $\operatorname{Pro}(\mathbf{1})$ is the entire class. The permutations 12 and 21 are self-prolific, but 1 is not prolific for either of them (since it has the other one as a one point extension). For permutations of size 3 or more, the prolific permutations, as in the previous subsection are the permutation of the same type (increasing or decreasing) of size at least one less than the size of the permutation.

\section{2 $\operatorname{Av}(321,231,132,213)$ and $\operatorname{Av}(321,231,132,312)$}

Both of these classes also have two permutations of each size greater than 1 .

The first class can be described as all the subpermutations of permutations in $\mathbf{1} \ominus \mathcal{I}$, while the second consists of all subpermutations of $21 \oplus \mathcal{I}$. 
In each case, a simple analysis using the ideas of the preceding subsection applies. Specifically, in the first case, for permutations of size at least 2 :

$$
\begin{aligned}
\operatorname{Pro}(21) & =\uparrow 21 \\
\operatorname{Pro}(12 \cdots k)=\operatorname{Pro}((k+1) 12 \cdots k) & =\uparrow k 12 \cdots(k-1) \quad(k \geqslant 2)
\end{aligned}
$$

In the second case:

$$
\begin{aligned}
\operatorname{Pro}(21) & =\emptyset \\
\operatorname{Pro}(12 \cdots k)=\operatorname{Pro}(213 \cdots k) & =\uparrow 213 \cdots(k-1) \quad(k \geqslant 2)
\end{aligned}
$$

\subsection{Non-Constant Polynomial Classes}

We now turn to classes with more relaxed restrictions that lead to slightly more interesting interpretations of prolificity.

These classes are

$$
\operatorname{Av}(132,213,321), \operatorname{Av}(312,231,213), \operatorname{Av}(321,231,132), \text { and } \operatorname{Av}(321,132)
$$

These permutation classes all have polynomial growth. Classes of this type are well understood - for instance Homberger and Vatter [5] showed that all such classes can be expressed as the union of grid classes for a finite set of peg permutations. In this instance, these classes correspond to the grid classes of the peg permutations

$$
2^{+} 1^{+}, \quad 1^{+} 2^{-}, \quad 2^{\bullet} 1^{+} 3^{+}, \text {and } 2^{+} 1^{+} 3^{+}
$$

respectively. However, rather than describing their notation in detail, it is easier to show the general shape of permutations in these classes:

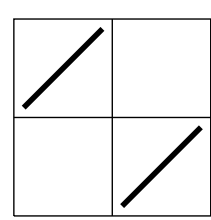

$2^{+} 1^{+}$

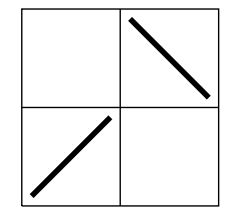

$1^{+} 2^{-}$

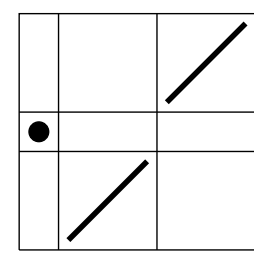

$2^{\bullet} 1^{+} 3^{+}$

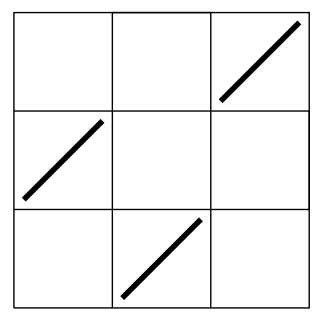

$2^{+} 1^{+} 3^{+}$

That is, the permutations in $2^{+} 1^{+}$are exactly those that can be divided from left to right into two increasing segments, the first of which lies above the second (and either could be empty). An alternative viewpoint is that they are those permutations that can be obtained from 21 by replacing either element by an increasing sequence of arbitrary length, maintaining the relative position of those two segments (and allowing either to be empty). Similarly, those in $2^{\bullet} 1^{+} 3+$ are obtained from 213 by replacing the 1 and 3 by increasing segments, while leaving 2 as a single point, or possibly deleting it.

The permutations in each class can be described by the number of elements belonging to each segment or dot, i.e., by a finite sequence of non-negative integers - for consistency we will list these from left to right, so for instance the pair $(3,2)$ describes the permutation 
34512 in $2^{+} 1^{+}$and the permutation 12354 in $1^{+} 2^{-}$, while the triple $(3,1,2)$ describes the permutation 234156 in $2^{+} 1^{+} 3^{+}$. We will identify a permutation with (any of) its representations in each case.

This representation makes it easy to describe and understand prolificity in these classes. Although quite similar, it is simplest to deal with each case in turn.

Proposition 10. Let $\pi=(a, b)$ be a permutation in $2^{+} 1^{+}$.

- If $a>0$ and $b>0$, then $\pi$ is self-prolific and is the minimal $\pi$-prolific permutation.

- If $a+b>0$, but $a=0$ or $b=0$ (i.e., $\pi$ is increasing), then there is a unique minimal $\pi$-prolific permutation described by the pair $(a+b-1, a+b-1)$.

Proof. Consider the first case and let $\tau=(c, d)$. If $c<a$, then the extension $(c, d+1)$ does not contain $\pi$, while if $d<b$ then the extension $(c+1, d)$ does not contain $\pi$. In either case, $\tau$ is not $\pi$-prolific. Conversely if $a \leqslant c$ and $b \leqslant d$ then $\pi \leqslant \tau$ and it is easy to see that every one-point extension of $\tau$ contains new copies of $\pi$ using that point.

In the second case, let $s=a+b$, so we also have $\pi=(s, 0)$ (or $(0, s))$. Suppose that $\tau=(c, d)$ with $c<s-1$ or $d<s-1$. Then, adding a point to the shorter of $\tau$ 's segments creates no copies of $\pi$. On the other hand, all extensions of $(s-1, s-1)$ create a copy of $\pi$ using the new point.

While this is the simplest of the four cases, the remaining arguments are all very similar so we shall be quite brief.

Proposition 11. Let $\pi=(a, b)$ be a permutation in $1^{+} 2^{-}$.

- If $a>0$ and $b>1$, then $\pi$ is self-prolific and is the minimal $\pi$-prolific permutation.

- If $\pi$ is decreasing and of size at least two, then $\operatorname{Pro}(\pi)=\{\}$.

- If $\pi$ is increasing, then then $\pi$ is self-prolific and is the minimal $\pi$-prolific permutation.

Proof. For the first case, that $\pi$ is self-prolific is clear. If $\tau$ does not contain $\pi$ then one of its segments is "too short", and lengthening the other one does not create any copies of $\pi$. For the second case, given any $\tau, 1 \oplus \tau$ is still in the class and contains no new copies of $\pi$. Finally for the third case, if $\tau$ does not contain $\pi$ then adding a new point which forms part of a proper decreasing segment creates no copy of $\pi$, while $\pi$ is clearly self-prolific since, even if we change the final element to a decreasing pair, we create a copy of $\pi$ using the newly added element.

Proposition 12. Let $\pi=(a, b, c)$ be a permutation in $2^{\bullet} 1^{+} 3^{+}$.

- If $a=1, b>0$, and $c>0$, then $\pi$ is self-prolific and is the minimal $\pi$-prolific permutation. 
- If $\pi$ is increasing of size $s$, then the minimal $\pi$-prolific permutations are represented by $(1, a, b)$ with $a+b=s-1$.

- If $\pi=1 \ominus \theta$, then $\operatorname{Pro}(\pi)=\{\}$.

Proof. In the first case, if $\tau=(s, t, u)$ does not contain $\pi$ then either $s=0, t<b$ or $u<c$. In each case, forming an extension by adding elements to some other segment forms no copies of $\pi$ at all. On the other hand, $\pi$ is easily seen to be self-prolific, since extending any segment (where possible) creates new copies of $\pi$.

If $\pi$ is increasing of size $s$ then no increasing permutation, $\tau$, is $\pi$-prolific since $1 \ominus \tau$ contains no new copies of $\pi$. Any $\pi$-prolific permutation must contain an increasing subsequence of size at least $s-1$ (or else, no one-point extension can contain a copy of $\pi$ ), so it must contain one of the permutations listed. But each of these is clearly $\pi$-prolific.

In the final case, note that for any $\tau, \tau \oplus 1$ is in the class and contains no new copies of $\pi$.

Proposition 13. Let $\pi=(a, b, c)$ be a permutation in $2^{+} 1^{+} 3^{+}$.

- If $a>0, b>0$, and $c>0$, then $\pi$ is self-prolific and is the minimal $\pi$-prolific permutation.

- If $\pi$ is increasing of size $s$, then the minimal $\pi$-prolific permutations are represented by $(a, a, b)$ with $a+b=s-1$.

- If $\pi$ contains 21 but not 213 , then $\operatorname{Pro}(\pi)=\{\}$.

Proof. These all correspond to the preceding proposition, except perhaps the second case, but if $\tau=(x, y, z)$ with $x+z<s-1$, then $(x+1, y, z)$ contains no new copies of $\pi$ and similarly if $y+z<s-1$. Conversely if both are at least $s-1$ then adding a point to any segment creates a new copy of $\pi$.

These propositions have analogous versions in any polynomial class, though the number of special cases can become quite large. Roughly speaking, if we have a maximal pegged permutation in the class and fill each segment (with one point for dots and two points for monotone segments) to create a permutation $\pi$, then we can guarantee that $\operatorname{Pro}(\pi)=\uparrow \pi$, while otherwise a more careful case analysis is required.

\subsection{Classes related to compositions}

The next group of classes we consider are the first that have exponential growth ratesthere are three, all closely related to the class of compositions that was considered in [3]. We extend some of the results of that paper here. The three classes are the following:

$$
\operatorname{Av}(231,312), \operatorname{Av}(312,321) \text {, and } \operatorname{Av}(231,312,321) \text {. }
$$

The first is the sum-closure of the class of decreasing permutations. Permutations in this class are also known as layered permutations. The second is the sum-closure of the 
class of cycles - permutations that are the skew-sum of an increasing permutation and 1 (i.e., of the form $23 \cdots k 1$ ). Since there is one decreasing permutation of each positive length, and one cycle of each positive length, the bijective correspondence between these classes and the class of compositions is clear - simply list the size of each sum-component.

As can be seen from the basis, the third class is the intersection of these two, so the sum-closure of 1 and 21. It is enumerated by the Fibonacci numbers.

With respect to prolificity, all three classes behave similarly. We'll consider the class of layered permutations in detail, and then indicate what variations occur in the other two examples. Here, we find it more convenient to work directly with the compositions that represent layered permutations rather than with the permutations themselves. The relevant ordering between compositions $u=u_{1} \cdots u_{k}$ and $v=v_{1} \cdots v_{n}$ is then that $u \leqslant v$ if and only if there exist $1 \leqslant i_{1}<\cdots<i_{k} \leqslant n$ with $u_{j} \leqslant v_{i_{j}}$ for $1 \leqslant j \leqslant k$. For compositions, we use the words prefix and suffix in the obvious way. To avoid triviality, compositions will be assumed to have sum greater than 1 unless that case is specifically mentioned. We first recall two results from [3]:

Theorem 14 (Theorem 3.1 of [3]). If $u=u_{1} \cdots u_{k}$, then $\operatorname{Pro}(u)=\{\}$ unless $u_{1}=u_{k}=1$.

Define a block to be a composition all of whose parts have size at least 2 . We will use Greek letters to denote blocks. For a block $\alpha=a_{1} \cdots a_{k}$ define:

$$
D(\alpha)=a_{1} \cdots a_{k-1} \max \left(a_{1}, a_{k}\right) a_{2} \cdots a_{k} .
$$

Note that if $\alpha$ is an empty block, then so is $D(\alpha)$, while if $\alpha$ is a block with one part, then $D(\alpha)=\alpha$.

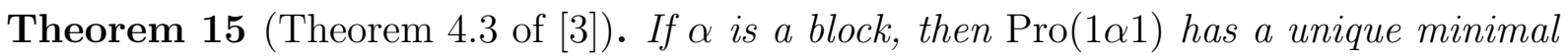
element which is $1 D(\alpha) 1$.

We now turn to some new results. Given a composition, $p$, of the form $1 \alpha_{1} 1 \alpha_{2} 1 \cdots 1 \alpha_{k} 1$ where each $\alpha_{i}$ is a (possibly empty) block, define:

$$
\mathcal{D}(p)=\left\{1 \alpha_{1} 1 \cdots \alpha_{i-1} 1 D\left(\alpha_{i}\right) 1 \alpha_{i+1} 1 \cdots \alpha_{k} 1: 1 \leqslant i \leqslant k\right\} .
$$

That is, $\mathcal{D}(p)$, is the set of compositions obtained from $p$ from replacing one of its maximal blocks with the result of applying $D$ to that block.

The main result of this section is:

Theorem 16. If a composition $p$ begins and ends with 1 then $t \in \operatorname{Pro}(p)$ if and only if every element of $\mathcal{D}(p)$ has an embedding in $t$.

Proof. Let $p$, beginning and ending with 1 be given, and let $\alpha=a_{1} a_{2} \ldots a_{k}$ be any non-trivial block of $p$ (i.e., $k>0$ ). Factor $p$ as $P \alpha S$ and let $t$ be any $p$-prolific word. Then, since increasing the size of the final part of $t$ by 1 must create new copies of $p$, we have that $p \leqslant t$ (Lemma 3.2 of [3]). Write $t=X Y$ where $X$ is the shortest prefix of $t$ containing $P a_{1} a_{2} \ldots a_{k-1}$. Now consider the extension $t^{+}=X 1 Y$ of $t$. Since $t$ is 
$p$-prolific, the designated 1 must occur in a copy of $p$. Since $P \alpha \nless X$, the corresponding 1 in $p$ must precede $\alpha$ and so $\alpha S \leqslant Y$. Consequently, $P a_{1} a_{2} \ldots a_{k-1} a_{1} a_{2} \ldots a_{k} S \leqslant t$. Arguing correspondingly from the shortest suffix of $t$ containing $a_{2} \ldots a_{k} S$, we also have $P a_{1} a_{2} \ldots a_{k} a_{2} \ldots a_{k} S \leqslant t$. Write $t=Q A R$ where $Q$ is the shortest prefix of $t$ containing $P a_{1} a_{2} \ldots a_{k-1}$ and $R$ its shortest suffix containing $a_{2} \ldots a_{k} S$. The two preceding conditions imply that $A$ contains both $a_{1}$ and $a_{k}$, and hence $\max \left(a_{1}, a_{k}\right)$, i.e., that $t$ contains $P D(\alpha) S$.

Conversely, suppose that every element of $\mathcal{D}(p)$ has an embedding in $t$. Then certainly $p \leqslant t$ so $t 1$ contains more copies of $p$ than $t$ does. Now consider any factorisation $t=X a Y$ and consider the two extensions $X 1 a Y$ and $X(a+1) Y$ of $t$. If $p$, with its final 1 deleted is contained in $X$ then both of these contain new copies of $p$. Otherwise, there is some block $\alpha$ of $p$, say $p=P 1 \alpha 1 S$ such that $P \leqslant X$ but $P 1 \alpha \nless X$. However, $P 1 D(\alpha) 1 S \leqslant t$. So, we can write $t=P^{\prime} M S^{\prime}$ where $P^{\prime}$ is the shortest prefix of $t$ containing $P$ and $S^{\prime}$ is the shortest suffix of $t$ containing $S$, and by the choice of $P$, the part $a$ belongs to $M$. Moreover $1 D(\alpha) 1 \leqslant M$, so $M$ is $1 \alpha 1$-prolific by Theorem 15. Therefore, both of the indicated extensions of $t$ contain a new copy of $1 \alpha 1$ within $M$, and hence a new copy of $p$. Thus, $t$ is $p$-prolific.

Recall that we have two classes of permutations that correspond bijectively to compositions (layered permutations and sums of cycles) and one that corresponds to compositions whose part sizes are at most 2. As the proof is written the preceding result applies only to the layered permutations. However, it is immediately clear that the bound on part sizes has no effect on the argument, so it applies to the last case as well.

The case of sums of cycles requires a little more consideration. The reason is that the ordering of the permutations is no longer the simple dominated subword ordering that we used. For instance the permutation 12 occurs as a pattern in 231 and so, in the corresponding compositions, we have $11 \leqslant 3$. However, the obvious generalisation of this is the only change to the ordering - runs of up to $k-11$ 's can be embedded into a single $k$ but single parts of size greater than 1 can only be embedded into single parts of the same size or greater. Since the first half of the proof of Theorem 16 uses only the greediness of being able to establish ordering, and the behaviour of the ordering on parts of size greater than 1 it remains valid. The second part of the proof effectively uses only greediness and Theorem 15 - which in turn, since it deals with compositions having only isolated parts of size one, covers this case as the underlying order is just the same for such compositions.

\section{5 $\operatorname{Av}(321)$}

Up to symmetry, the only remaining class of permutations having basis elements of size three or less with a non-trivial notion of prolificity is $\operatorname{Av}(321)$. The underlying partially ordered set of containments between permutations in this class is very complex - for instance, it contains infinite antichains. It's therefore unsurprising that this class presents significantly more difficulty in describing sets of texts $t$ that are prolific for a pattern $p$ in any generality, and we present here only some initial observations. The real difficulty arises from the fact that our previous techniques required one of two conditions to hold: a structural characterisation of all permutations in the class based on a bounded number of 


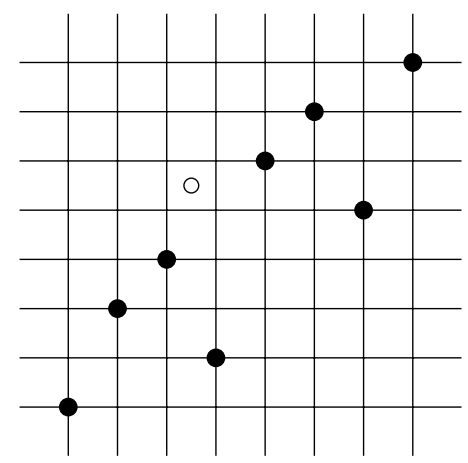

Figure 2: The indicated point extends $1 \oplus 231 \oplus 231 \oplus 1$ inside $\operatorname{Av}(321)$ without creating a new copy of $1 \oplus 231 \oplus 1$.

integer parameters, or, a correspondence with compositions (and words more generally) with a simple relationship between the containment ordering in permutations and some sort of "dominated-subword" ordering on the words.

In $\mathrm{Av}(321)$, as in any sum-closed class, we can represent the permutations as words over the alphabet of sum-indecomposable permutations. However, the containment relationship does not interact smoothly with this representation. In particular, it is easy to find examples of the form $\alpha \oplus \beta \leqslant \gamma$, where all three permutations are sum-indecomposable. For instance $231 \oplus 312 \leqslant 2351746$. Even more generally, every permutation in $\operatorname{Av}(321)$ can be embedded into a sum-indecomposable one. For 321-avoiding permutations without fixed points (i.e., sum-indecomposable components of size one), there is a form of greedy embedding (see $[1,2]$ ), but this is of little utility for studying prolificity.

As always, we note that if the first sum-component of a permutation $\pi \in \operatorname{Av}(321)$ is not 1 , then $\operatorname{Pro}(\pi)=\{\}$ as, for any $\tau, 1 \oplus \tau$ contains no more copies of $\pi$ than $\tau$ did. Similarly, if the last sum-component is not 1 then $\operatorname{Pro}(\pi)=\{\}$. Of course, if $\pi=1$ then $\operatorname{Pro}(\pi)=\operatorname{Av}(321)$.

In the case of layered permutations, those permutations that were of the form $1 \oplus k \oplus 1$ for some integer $k$ were self prolific. In $\operatorname{Av}(321)$ there is no permutation $\alpha$ for which $1 \oplus \alpha \oplus 1$ is self-prolific.

Proposition 17. The only self-prolific permutation in $\operatorname{Av}(321)$ is 1.

Proof. Let $\pi=1 \oplus \alpha \oplus 1$. Consider inserting a new element to the left of $\pi$ lying above the two least elements of $\pi$. This creates no copies of 321 since the two least elements of $\pi$ occur in increasing order. The only role that the new element could play in a copy of $\pi$ would be as the leftmost 1 , but there are too few elements that lie above it for this to be possible.

Pursuing the analogy with layered permutations a bit farther, in that case it was true that $1 \oplus \pi \oplus \pi \oplus 1$ was always $(1 \oplus \pi \oplus 1)$-prolific. Figure 2 shows that this fails in $\operatorname{Av}(321)$. However, if we add a bit of padding in the form of an extra 21 summand between the two copies of $\pi$ we do recover prolificity. 


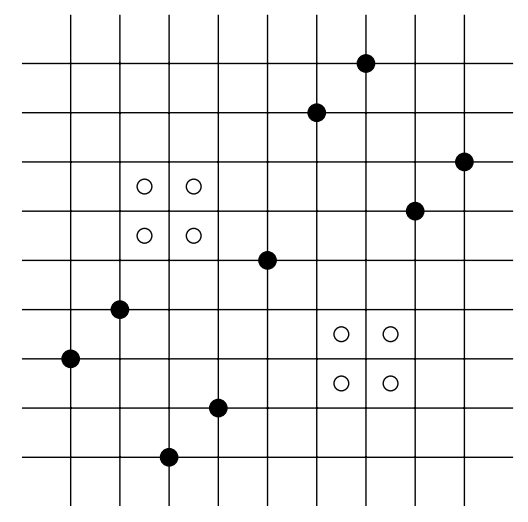

Figure 3: Eight extensions of 241358967 used to build an example of a permutation $\pi$ for which $1 \oplus \pi \oplus 1 \oplus \pi \oplus 1$ is prolific for $1 \oplus \pi \oplus 1$, but $1 \oplus \pi \oplus \pi \oplus 1$ is not.

Proposition 18. For any permutation $\pi \in \operatorname{Av}(321), 1 \oplus \pi \oplus 21 \oplus \pi \oplus 1$ is prolific for $1 \oplus \pi \oplus 1$.

Proof. Consider any one-point extension of $1 \oplus \pi \oplus 21 \oplus \pi \oplus 1$. If the new point lies to the left of the 2, it must lie below it (else a 321 is formed) and so can play the role of the first 1 in a copy of $1 \oplus \pi \oplus 1$. Similarly, if it lies to the right of the 1 it must lie above it and can play the role of the last 1 in a copy of $1 \oplus \pi \oplus 1$. If it lies between the 2 and the 1 in position, it cannot also lie between them in value and so can play either the role of the first or last 1 in a copy of $1 \oplus \pi \oplus 1$ depending on whether it lies below the 1 , or above the 2 .

There do exist non-trivial permutations $\pi \in \operatorname{Av}(321)$ for which $1 \oplus \pi \oplus \pi \oplus 1$ is $1 \oplus \pi \oplus 1$ prolific. A large group of these arise when $\pi$ has its maximum as its second last element, and its minimum as its second element. Suppose that is the case and consider a one point extension of $1 \oplus \pi \oplus \pi \oplus 1$. If the new point lies before and below the second copy of $\pi$ or above and after the first copy then we have created a new copy of $1 \oplus \pi \oplus 1$. If it lies above, but not after, the first copy of $\pi$ then it cannot lie before the second last element of that copy (or else a 321 would be created). In that case, it can play the role of the maximum element of a copy of $\pi$ (basically just replacing the maximum in the original first copy) as part of a copy of $1 \oplus \pi \oplus 1$. Similarly, if it lies below, but not before, the second copy of $\pi$ it can play the role of a 1 within that copy of $\pi$. There are other similar criteria relating to the last element of $\pi$ being its second-greatest, or the first element being its second smallest. Finally, there is a group of permutations, the increasing oscillations of even length, to which these criteria do not apply directly, but which none the less have the property that $1 \oplus \pi \oplus \pi \oplus 1$ is prolific for $1 \oplus \pi \oplus 1$. The authors had hoped this might be a complete characterisation of permutations with that property but a counterexample ${ }^{1}$ was provided by Robert Brignall (personal communication, September 11, 2020).

Finally, in this vein, let us consider whether we can find $\pi \in \operatorname{Av}(321)$ such that $1 \oplus \pi \oplus \pi \oplus 1$ is not prolific for $1 \oplus \pi \oplus 1$ but $1 \oplus \pi \oplus 1 \oplus \pi \oplus 1$ is prolific for $1 \oplus \pi \oplus 1$.

\footnotetext{
${ }^{1}$ Let $\pi=316284510712914111713191516211820.1 \oplus \pi \oplus \pi \oplus 1$ is $1 \oplus \pi \oplus 1$-prolific.
} 
In Figure 3 we describe eight permutations that can be formed by extending 241358967 by a single element. Call these $\alpha_{1}$ through $\alpha_{8}$ (the order doesn't matter) and take $\pi$ to be $\alpha_{1} \oplus \alpha_{2} \oplus \cdots \oplus \alpha_{8}$. Now, in $1 \oplus \pi \oplus \pi \oplus 1$ we can add a single point that ties together the $\alpha_{8}$ of the first $\pi$ to the $\alpha_{1}$ of the second in such a way that no new copies of $1 \oplus \pi \oplus 1$ are created. Basically, in the inversion graph we add a cut vertex of degree two - as there are no such vertices in any $\alpha_{i}$ and as it can't play the role of a 1 , we can't create a new copy of $1 \oplus \pi \oplus 1$. On the other hand, in a one-point extension of $1 \oplus \pi \oplus 1 \oplus \pi \oplus 1$, if the new point is before and below the second $\pi$ or above and after the first, we have a new copy of $1 \oplus \pi \oplus 1$. There are eight remaining places where an extension could be made (tying together $\alpha_{8}$ and $\alpha_{1}$ as before, but now around the interposing 1). Each of these creates a copy of some $\alpha_{i}$ using the last four elements from $\alpha_{8}$, the interposing 1 , and the first four elements from $\alpha_{1}$. So we get a new copy of $1 \oplus \pi \oplus 1$ by using the original $1, \alpha_{1}$ through $\alpha_{i-1}$ from the first copy of $\pi$, the newly created $\alpha_{i}$ and $\alpha_{i+1}$ through $\alpha_{8}$ from the second copy of $\pi$. Note that in this example, $\pi$ is of size 80-we're confident that smaller examples exist!

An obvious question to ask is: what are the prolific permutations for $\pi=12 \cdots \mathrm{s}$ $(s \geqslant 2)$ in $\operatorname{Av}(321) ?$

The case $\pi=12$ is easy to resolve. No increasing permutation is prolific for 12 (since we could add a new maximum at the beginning, or a new minimum at the end without creating any new copies of 12), while every permutation in $\operatorname{Av}(321)$ that contains 21 is prolific, since any added point must form an increasing pair with at least one point in any copy of 12 .

However, even $\pi=123$ is already somewhat more complicated. The minimal prolific permutations for 123 in $\mathrm{Av}(321)$ are:

$$
1324,2143,2413,3142,3412 \text {. }
$$

The non-123-prolific permutations are all of those that are the sum of an increasing permutation and a single cycle (i.e., a permutation in $1 \ominus \mathcal{I}$ or $\mathcal{I} \ominus 1$ ). Another way to think of these is that they are formed from an increasing permutation by adding a single point outside the bounding box formed by the increase. It is easy to verify that these are non-prolific - for instance those obtained from an increasing permutation by adding a point to the right can be extended by a new minimum in the second last position without creating a copy of 123 .

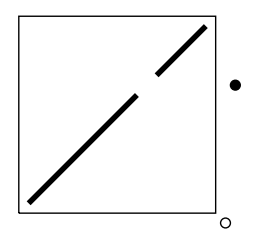

Since it is easily checked that the permutations in $\operatorname{Av}(321)$ that also avoid the five listed permutations above are exactly the ones of this type, that establishes that the 123-prolific permutations in $\operatorname{Av}(321)$ are exactly those that involve one of the five listed permutations above.

Beyond $\pi=123$ things become more complicated. It appears that there are 44 minimal 1234-prolific permutations all of size six. It also appears that there are 453 minimal 12345- 
prolific permutations, one of size seven (1324657) and the rest of size eight. In both cases there are no further minimal prolific permutations of size 14 or less. Also in both cases the subclass of $\operatorname{Av}(321)$ avoiding these permutations is of polynomial growth. Using results from [6], that last observation is at least one we can prove.

Theorem 19. The non-prolific permutations in $\mathrm{Av}(321)$ for $12 \cdots s$ form a subclass of polynomial growth.

Proof. A necessary and sufficient condition for a subclass of $\operatorname{Av}(321)$ to be of polynomial growth (combining Theorem 2.5 and Proposition 3.2 from [6] and taking into account that we already avoid 321) is that, for some $k$ it contains none of the three permutations:

$$
2143 \cdots 2 k(2 k-1), \quad 246 \cdots(2 k) 135 \cdots(2 k-1), \quad(k+1) 1(k+2) 2 \cdots(2 k) k .
$$

Note that for $k=s-1$ the Robinson-Schensted tableau for each of these three permutations is a $2 \times(s-1)$ rectangle. Adding any single element while still avoiding 321 (hence not creating a third row of the tableau) creates a first row of size $s$, and therefore an increasing subsequence of that size which must necessarily use the new element. In other words, each of these three permutations is $12 \cdots s$ prolific and so the non-prolific permutations for $12 \cdots s$ in $\mathrm{Av}(321)$ form a class with polynomial growth.

\section{Conclusion}

It seems to us that the notion of prolificity is a natural one that would repay further investigation. Of course, in some contexts (where many extensions or very few exist) it is somewhat trivial. But, there seem to be a large variety of combinatorial classes where it is non-trivial, and often in an interesting way.

\section{Acknowledgements}

We would like to thank Dr Robert Brignall (The Open University), Dr Dominic Searles (University of Otago), and Dr Christopher Tuffley (Massey University) for their input during the examination of the thesis from which this work was derived, and Dr Vincent Vatter (University of Florida) for reminding us of the necessary and sufficient characterisation of polynomial growth in [6].

\section{References}

[1] M.H. Albert, M.D. Atkinson, R. Brignall, N. Ruškuc, Rebecca Smith and J. West, Growth rates for subclasses of $\operatorname{Av}(321)$, Electron. J. Combin., 17, \#R141, 2010.

[2] M. Albert, M.-L. Lackner, M. Lackner and V. Vatter, The complexity of pattern matching for 321-avoiding and skew-merged permutations. Discrete Math. Theor. Comput. Sci. 18, no. 2, Paper No. 11, 17 pp, 2016. 
[3] M. Albert and M. Tannock, Prolific Compositions. Discret. Math. Theor. Comput. Sci., 21(2), 2019.

[4] D. Bevan, C. Homberger and B.E. Tenner, Prolific Permutations and Permuted Packings: Downsets Containing Many Large Pattern J. Combin. Theory Ser. A, 153:98-121, 2018.

[5] C. Homberger and V. Vatter, On the Effective and Automatic Enumeration of Polynomial Permutation Classes. J. Symb. Comput., 76:84-96, 2016.

[6] S. Huczynska and V. Vatter, Grid Classes and the Fibonacci Dichotomy for Restricted Permutations Electron. J. Combin., 13, \#R54, 2006.

[7] R. Simion and F. W. Schmidt, Restricted Permutations. Eur. J. Comb., 6(4):383406, 1985.

[8] M. Tannock, Prolific Structures in Combinatorial Classes (Thesis, Doctor of Philosophy). University of Otago, 2020. 